\title{
Imaging of immunotherapy response in non-small cell lung cancer: challenges and perspectives
}

\author{
Andrea Ciarmiello ${ }^{1} \cdot$ Rosa Fonti $^{2} \cdot$ Giampiero Giovacchini $^{1} \cdot$ Silvana Del Vecchio ${ }^{3}$
}

Published online: 29 November 2018

(c) Italian Association of Nuclear Medicine and Molecular Imaging 2018

Immunotherapy has been recently included as new therapeutic option, in addition to conventional and targeted therapy, for the treatment of non-small cell lung cancer (NSCLC) patients. The advent of this approach, targeting the host immune system rather than cancer cells, has caused a paradigm shift in cancer treatment. Cancer cells can evade immune surveillance by inhibiting the native capacity of immune cells to recognize, and destroy abnormal and foreign cells. Immunotherapy mobilizes the immune system by restoring the ability of immune cells to mount an effective immune response against tumor cells.

$\mathrm{T}$ cell activity can be regulated by a number of factors including modulation of inhibitory immune checkpoints. These are a large class of co-receptors expressed by $\mathrm{T}$ cells that negatively regulate $\mathrm{T}$-cell response to antigen thus promoting self-tolerance and preventing autoimmunity. Specific monoclonal antibodies can bind to these co-receptors suppressing the negative feedback and inducing an activation of immune cells against cancer cells. The immune checkpoints currently used as targets for blockade therapy in cancer patients include programmed death-1 (PD-1), programmed death-1 ligand (PD-L1), cytotoxic T-lymphocyte antigen 4 (CTLA-4) and lymphocytes antigen gene 3 (LAG-3). Clinical trials in metastatic melanoma and non-small cell lung cancer showed durable responses and increased overall survival in patients treated with immunotherapy. Nivolumab and pembrolizumab are anti-PD-1 monoclonal antibodies approved by FDA and EMA as second-line therapy in advanced NSCLC. In addition, pembrolizumab can be used

Andrea Ciarmiello

andrea.ciarmiello@as15.liguria.it

1 Department of Nuclear Medicine, S. Andrea Hospital, 19124 La Spezia, Italy

2 Institute of Biostructures and Bioimages, National Research Council, Naples, Italy

3 Department of Advanced Biomedical Sciences, University of Naples Federico II, Naples, Italy as first-line therapy in patients whose tumors have a high PD-L1 expression ( $\geq 50 \%$ ), and are negative for EGFR mutations and ALK rearrangements. Atezolizumab is an anti-PD-L1 monoclonal antibody approved for the treatment of metastatic NSCLC refractory to first-line platinum-based regimens.

Despite the improvement of clinical outcome of NSCLC patients using these agents, several issues need to be clarified especially in response assessment at early time points. Clinical experience with these immunomodulatory monoclonal antibodies, in fact, revealed that several patterns of response to immunotherapies fail to be adequately recognized by international response criteria such as WHO and RECIST. Therefore, adaptation of these criteria leads to the development of immune-related response criteria (irRC) and immune-related RECIST (irRECIST) [1]. These adapted criteria took into account some peculiar aspects of immunotherapy response: (1) reduction of tumor burden in response to immunotherapy may be detected at later time points as compared to that induced by cytotoxic chemotherapy; (2) the increase of inflammatory cells recruited within the tumor may induce an apparent paradoxical increase in size of the tumor lesion ("pseudoprogression"); (3) the appearance of a new lesion does not necessarily represent progression of the disease and may not require discontinuation of immunotherapy.

Another debated issue in the use of immunomodulatory monoclonal antibodies in NSCLC patients was related to the identification of clinical characteristics, biological or circulating markers that may predict which patient can benefit from immunotherapy. Currently, PD-L1 status is the selection criterion for the use of pembrolizumab in NSCLC patients, since high levels of PD-L1 were associated with improved survival of patients treated with this monoclonal antibody. The predictive role of PD-L1 expression was less univocally defined for patients treated with nivolumab since a survival benefit was found in PD-L1-positive patients with a non-squamous histology, whereas in patients with 
squamous cell carcinoma the outcome was independent from PD-L1 status. Although atezolizumab recognizes PD-L1, the survival benefit of patients treated with this monoclonal antibody was found in both PD-L1-positive and -negative tumors.

In this complex clinical scenario, another matter of discussion is whether functional imaging with ${ }^{18} \mathrm{~F}$-FDG PET$\mathrm{CT}$ can be of aid to monitor response to immunotherapy and to select patients with NSCLC. Since effects of immunotherapy occur later than those induced by conventional chemotherapy, the first issue to be addressed is the optimal timing of imaging. In NSCLC patients, according to RECIST, an objective response to PD1/PD-L1 inhibitors was reported to occur approximately 2 months after the beginning of therapy. Therefore after a baseline ${ }^{18}$ F-FDG PET-CT scan, it would be advisable to perform the first follow-up study not earlier than 2 months. Since metabolic changes usually precede anatomic response to anti-cancer treatment, at this time point metabolic response is expected to be more pronounced than morphological changes. However, both functional and anatomical imaging can be affected by the phenomenon of pseudoprogression. In fact, lymphocytic infiltration and inflammation, in addition to an increase of tumor size, may cause an enhanced ${ }^{18} \mathrm{~F}$-FDG uptake or possible appearance of new FDG-avid lesions, otherwise too small to be detected. Notably, in NSCLC patients, pseudoprogression occurs with a lower frequency than in melanoma, namely $0.6-5 \%$ vs 4-10\% [2]. Therefore, this phenomenon can be less detrimental in NSCLC than in melanoma patients undergoing ${ }^{18}$ F-FDG PET-CT imaging. However, in patients showing an increase of tumor size and/or ${ }^{18} \mathrm{~F}$-FDG uptake at the first scan after the beginning of immunotherapy, a second followup scan at 4-8 weeks is recommended to confirm or not disease progression. Furthermore in responding patients, the enhancement of FDG uptake in bowel, pancreas, thyroid, adrenals, or pituitary can be due to the occurrence of immune-related adverse effects.

Recent studies evaluated the use of ${ }^{18}$ F-FDG PET-CT for the selection of NSCLC patients who can benefit from immunotherapy. A high ${ }^{18} \mathrm{~F}$-FDG uptake in NSCLC patients was reported to be associated with high levels of PD-1 [3] and PD-L1 [4] in tumors suggesting that ${ }^{18}$ F-FDG PET-CT could be used to identify potential responders to immunotherapy. While contrasting results were reported on the predictive value of PD-L1, ${ }^{18}$ F-FDG uptake at baseline [3] and after 1 month immunotherapy [5] was found to be predictive of clinical outcome. However, the predictive role of ${ }^{18}$ F-FDG PET-CT needs to be confirmed in larger clinical studies.

Another issue that needs to be dealt with is related to the use of non-FDG tracers. In particular, monoclonal antibodies recognizing PD-1 or PD-L1 have been labeled with $\left[{ }^{64} \mathrm{Cu}\right]$ or $\left[{ }^{89} \mathrm{Zr}\right]$ and tested in animal models of lung cancer. Although remarkable results were achieved in the characterization of these radiolabeled monoclonal antibodies in preclinical imaging studies, their transfer into a clinical context poses several challenges. In fact, the wide expression of inhibitory immune receptors and ligands in normal tissues, and not only in tumors may cause a high background but also in normal lymphoid organs. Furthermore, the development of animal models for imaging of immunotherapy response still relies on the use of immunocompetent mice and this implies the transplantation of murine tumor cell lines and the use of species-specific monoclonal antibodies against murine PD1/PD-L1.

In summary, while studies have already shown with convincing evidence the clinical efficacy of checkpoint inhibitors, an optimal imaging methodology for monitoring response to these drugs still needs to be identified. However, despite the pitfalls of pseudoprogression and the peculiar patterns of immunotherapy response, ${ }^{18} \mathrm{~F}-\mathrm{FDG}$ PET-CT still remains the primary imaging modality for the evaluation of tumor response also to immunomodulatory agents in NSCLC patients. Further studies are needed for optimization of imaging protocols in terms of timing and validation of the best metabolic parameter reflecting immunotherapy response to improve the management of these patients.

Funding None.

\section{Compliance with ethical standards}

Conflict of interest All authors declare that they have no conflict of interest.

Human/animal participants This article does not contain any studies with human or animal subjects performed by any of the authors.

\section{References}

1. Chiou VL, Burotto M (2015) Pseudoprogression and immunerelated response in solid tumors. J Clin Oncol 33:3541-3543. https://doi.org/10.1200/JCO.2015.61.6870

2. Nishino M, Dahlberg SE, Adeni AE, Lydon CA, Hatabu H, Janne PA et al (2017) Tumor response dynamics of advanced non-small cell lung cancer patients treated with PD-1 Inhibitors: imaging markers for treatment outcome. Clin Cancer Res 23:5737-5744. https://doi.org/10.1158/1078-0432.CCR-17-1434

3. Lopci E, Toschi L, Grizzi F, Rahal D, Olivari L, Castino GF et al (2016) Correlation of metabolic information on FDG-PET with tissue expression of immune markers in patients with non-small cell lung cancer (NSCLC) who are candidates for upfront surgery. Eur J Nucl Med Mol Imaging. 43:1954-1961. https://doi. org/10.1007/s00259-016-3425-2

4. Takada K, Toyokawa G, Okamoto T, Baba S, Kozuma Y, Matsubara T et al (2017) Metabolic characteristics of programmed cell death-ligand 1-expressing lung cancer on (18) 
F-fluorodeoxyglucose positron emission tomography/computed tomography. Cancer Med 6:2552-2561. https://doi.org/10.1002/ cam4.1215

5. Kaira K, Higuchi T, Naruse I, Arisaka Y, Tokue A, Altan B et al (2018) Metabolic activity by (18)F-FDG-PET/CT is predictive of early response after nivolumab in previously treated NSCLC. Eur J Nucl Med Mol Imaging. 45:56-66. https://doi.org/10.1007/ s00259-017-3806-1 